\title{
PERCEPCIÓN DE LOS BENEFICIOS DE LA TENENCIA DE ANIMALES DE COMPAÑÍA EN TRES GRUPOS POBLACIONALES DE LA HABANA, CUBA
}

\section{Perception of the Benefits of the OWnership of Companion Animals in Three Population Groups in Havana, Cuba}

\author{
Beatriz Hugues H. ${ }^{1,2}$, Aimee Álvarez A. ${ }^{1}$, Lizet Castelo E.C. ${ }^{1}$, Loraine Ledón L. ${ }^{1}$,

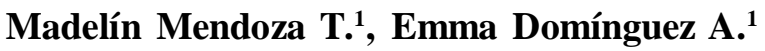

\section{Resumen}

El estudio tuvo como objetivo conocer la percepción de las personas de tres grupos poblacionales de La Habana, Cuba [Criadores de aves ornamentales (CAO), estudiantes de quinto año de la carrera de Medicina Veterinaria (E) y responsables de animales de compañía (RAC)] a través de una encuesta con respecto a la tenencia de animales de compañía y su relación con la satisfacción personal y la salud. El 99\% de las personas admitieron que se sentían muy satisfechas o satisfechas al compartir sus vidas con estos animales. En relación a la salud psicoemocional indicaron que les resultaba muy beneficiosa (84, 73 y $80 \%$ para CAO, E y RAC, respectivamente). Con relación al control metabólico, el 58 y $96 \%$ de personas en CAO y RAC que sufren de estos problemas señalaron que los motivaba a cuidarse más. Los resultados demuestran que las personas encuestadas en los tres grupos poblacionales perciben que la tenencia de animales de compañía les aporta beneficios a la salud física y mental.

Palabras clave: satisfacción personal, salud psicoemocional, control metabólico, actividades físicas, animales de compañía

\section{Abstract}

The study aimed to determine the perception of three groups of people in Havana, Cuba [breeders of ornamental birds (CAO), veterinary medicine students (E), and pet owners (RAC)] through a survey on pet ownership in relation to personal satisfaction and health. Results showed that $99 \%$ of the persons were satisfied of sharing their lives

\footnotetext{
${ }^{1}$ Instituto Nacional de Endocrinología (INEN), La Habana, Cuba

${ }^{2}$ E-mail: bettymig@infomed.sld.cu
}

Recibido: 5 de diciembre de 2013

Aceptado para publicación: 7 de abril de 2014 
with companion animals. They also pointed out high mental health benefits $(84,73$, and $80 \%$ for CAO, E, and RAC respectively). In relation to metabolic control, 58 and $96 \%$ of the persons suffering any of these diseases indicated that were motivated to take better care. The results showed that the surveyed persons in the three population groups perceive that pet ownership brings them physical and mental health benefits.

Key words: personal satisfaction, psicoemotional health, metabolic control, physical activities, companion animals

\section{INTRODUCCIÓN}

Los animales de compañía han desempeñado un rol preponderante en la vida de los seres humanos a través de la historia, proporcionando compañía, motivación y agrado, además de ejercer efectos positivos en la prevención y recuperación de la salud física y mental de las personas con quienes conviven o se vinculan (Mentzel, 2004).

El valor o significado de un animal de compañía está relacionado con factores tales como la edad y el sexo de la persona con la que se vincula. Los niños pequeños desarrollan sobre todo una relación de juegos, mientras que para los adolescentes tiene mayormente un significado de compañero, de protección y de mediador en la vida social (Cusack, 1991). Asimismo, en personas de la tercera edad, la convivencia con animales de compañía ayuda a elevar su calidad de vida, ya que por sus requerimientos de amor y cuidados pasan a ocupar un papel central en sus vidas (Gatti, 1994). Por otro lado, se han encontrado diferencias de género frente a la función que representaba poseer un animal de compañía, pues mientras que para las mujeres representan un facilitador social, incluyendo la ayuda para superar tiempos difíciles, los hombres consideran razones prácticas tales como facilitar el ejercicio físico o cumplir una función útil (Staats et al., 2006).

La convivencia con animales de compañía se ha ido incrementando en Cuba a partir de la década del 80 (W. Hernández, La
Habana, comunicación personal), lo cual ha adquirido una connotación social. Sin embargo, son pocos los estudios que se han realizado sobre la caracterización de la tenencia de animales de compañía en el medio (Beovides et al., 2011; Domínguez et al., 2011). Es así que se desarrolló el presente estudio, con el objetivo de conocer la percepción de tres grupos humanos de la población de La Habana, con respecto a la tenencia de animales de compañía y su relación con la satisfacción personal, beneficios sobre la salud mental y control metabólico, así como en la motivación para la realización de actividades físicas.

\section{Materiales y Métodos}

Se realizó un estudio descriptivo transversal, utilizando metodología cuantitativa de investigación, durante los meses de julio a septiembre de 2012.

La población objeto de estudio estuvo constituida por 115 personas responsables de animales de compañía (Grupo RAC) que solicitaron los servicios de consulta oftalmológica de la clínica veterinaria «José Luis Callejas» de La Habana; por 15 estudiantes (Grupo E) de quinto año de la Facultad de Medicina Veterinaria de la Universidad Agraria de La Habana "Fructuoso Rodríguez Pérez", quienes estaban realizando una pasantía en la clínica; y por 25 personas que se dedicaban a la cría de aves ornamentales (Grupo CAO) y cuyos animales recibieron atención en una 
clínica especializada de aves ornamentales de La Habana. El total de la población en estudio fue, por lo tanto, de 155 personas.

Como criterios de inclusión se consideraron hombres y mujeres, mayores de 18 años que tuvieran a su cargo animales de compañía, y como criterios de exclusión a personas con condiciones físicas o de salud mental que les dificultaba la lectura y comprensión del cuestionario del estudio.

El cuestionario sobre tenencia de animales de compañía comprendió 19 ítems que incluyeron los siguientes aspectos:

- Datos demográficos de las personas: género, edad, municipio de residencia, nivel de escolaridad, ocupación y convivencia o no con otras personas.

- Satisfacción personal sobre la tenencia de animales de compañía: Valoración de la satisfacción que siente una persona al estar en contacto directo con un animal de compañía, el bienestar que le produce esta interrelación. En el tema de la 'relación con el animal de compañía', los encuestados podían seleccionar más de una respuesta.

- Beneficios sobre la salud psicoemocional: Valoración de los posibles efectos beneficiosos que ejerce la tenencia de animales de compañía relacionados con los aspectos de la salud sicoemocional y la calidad de vida de las personas a su cargo.

- Beneficios sobre el control metabólico: Valoración de los posibles efectos beneficiosos que ejerce la tenencia de animales de compañía sobre el control metabólico de las personas que los atienden. Este aspecto solo fue valorado en las personas que padecían enfermedades endocrinas o metabólicas, como diabetes mellitus u otras como dislipidemias, en las cuales puede existir descontrol del metabolismo.

- Motivaciones para la actividad física: Se entendieron como tales las razo- nes que impulsaron la realización de actividades físicas (caminatas) en relación con la tenencia de perros como animales de compañía. Se tuvo en cuenta, además, la frecuencia de realización de estas caminatas. En la pregunta sobre el efecto positivo de las caminatas, el encuestado podía tener más de una respuesta.

La participación de los sujetos en el estudio fue voluntaria y anónima. A cada persona que cumplió con los criterios de inclusión se le solicitó su participación en el estudio, previa información de las características del estudio. Al consentir su participación se le brindó el cuestionario. Toda la información recogida se manejó de forma confidencial. No se recolectaron datos de identidad personal y los datos obtenidos fueron codificados y analizados mediante estadística descriptiva.

\section{Resultados y Discusión}

\section{Datos Demográficos}

Los datos demográficos se muestran en el Cuadro 1. Se observó una mayor presencia de mujeres, con excepción del grupo de estudiantes donde eran mayormente varones. En relación a la edad, la tenencia de animales de compañía predominó en el grupo de 31 a 40 años (30\%), en tanto que la tenencia de aves ornamentales predominó en el grupo de 21 a 30 años (32\%). La edad en el grupo de estudiantes estuvo obviamente sesgada (100\% entre 21 a 30 años).

Las personas que acudieron al servicio oftalmológico no procedían de un municipio en particular. Los resultados muestran una amplia variabilidad por municipio de residencia, tanto dentro de grupos como entre grupos. Cabe mencionar que si bien la clínica oftalmológica es de referencia nacional, la mayor proporción de pacientes proviene de los distritos cercanos. 
Cuadro 1. Datos demográficos de los grupos poblacionales encuestados (distribución porcentual)

\begin{tabular}{|c|c|c|c|c|}
\hline \multirow[b]{2}{*}{ Variable } & & \multicolumn{3}{|c|}{ Grupo poblacional } \\
\hline & & $\begin{array}{c}\text { Criadores de } \\
\text { aves } \\
\text { ornamentales } \\
(n=25)\end{array}$ & $\begin{array}{c}\text { Estudiantes } \\
\text { de Medicina } \\
\text { Veterinaria } \\
(\mathrm{n}=15)\end{array}$ & $\begin{array}{l}\text { Responsables de } \\
\text { animales de } \\
\text { compañía } \\
(\mathrm{n}=115)\end{array}$ \\
\hline \multirow[t]{2}{*}{ Género } & Femenino & 56 & 40 & 65 \\
\hline & Masculino & 44 & 60 & 35 \\
\hline \multirow{6}{*}{$\begin{array}{l}\text { Edad } \\
\text { (años) }\end{array}$} & $11-20$ & 12 & - & 3 \\
\hline & $21-30$ & 32 & 100 & 19 \\
\hline & $31-40$ & 16 & - & 30 \\
\hline & $41-50$ & 20 & - & 22 \\
\hline & $51-60$ & 8 & - & 10 \\
\hline & $>61$ & 12 & - & 16 \\
\hline \multirow{7}{*}{$\begin{array}{l}\text { Municipio de } \\
\text { residencia }\end{array}$} & Centro Habana & 12 & 13 & 12 \\
\hline & Habana V ieja & 12 & - & 10 \\
\hline & Cerro & 4 & - & 17 \\
\hline & Diez de Octubre & 8 & - & 10 \\
\hline & Habana del Este & 4 & 20 & 5 \\
\hline & Plaza de la Revolución & 8 & - & 14 \\
\hline & Otros & 48 & 54 & 10 \\
\hline \multirow{4}{*}{$\begin{array}{l}\text { Nivel de } \\
\text { escolaridad }\end{array}$} & Primaria & - & - & 5 \\
\hline & Secundaria & 4 & - & 9 \\
\hline & Medio & 52 & - & 42 \\
\hline & Superior & 44 & 100 & 43 \\
\hline \multirow[t]{3}{*}{ Ocupación } & $\begin{array}{l}\text { Amas de casa y } \\
\text { jubilados }\end{array}$ & 20 & - & 16 \\
\hline & Estudiantes & 4 & 100 & 10 \\
\hline & Trabajadores & 76 & - & 74 \\
\hline \multirow{2}{*}{$\begin{array}{l}\text { Convivencia } \\
\text { con otras } \\
\text { personas }\end{array}$} & Sí & 100 & 100 & 90 \\
\hline & No & - & - & 10 \\
\hline
\end{tabular}

El nivel de escolaridad de las personas entrevistadas fue notoriamente mayor en el nivel medio (preuniversitario) y universitario. Con relación a la ocupación laboral, la proporción de personas que trabaja fue similar y mayoritaria, tanto en el grupo RAC (74\%) como en el grupo CAO (76\%). Asimismo, el número de personas que vivía únicamente con sus animales se observó en muy baja frecuencia y solo en el grupo RAC.

Los autores no han encontrado referencias de estudios similares en La Habana, por lo cual no pueden comparar sus resultados 
con otros efectuados en esta provincia. No obstante, Beovides et al. (2011) realizaron una evaluación del cuidado de los animales de compañía en Santa Clara, Cuba, determinando que la población estaba compuesta sobre todo por hombres (53.3\%), con edades entre 18 a 30 años $(42.7 \%)$ y de 31 a 50 años (26.7\%), y especialmente con un nivel de escolaridad medio (59.3\%). Esta información difiere en relación al género con los datos del presente estudio.

\section{Satisfacción Personal}

Los resultados de la evaluación de la satisfacción personal con respecto a la tenencia de animales de compañía se encuentran en el Cuadro 2.

El 56\% de criadores de aves ornamentales tenían, además, otros animales, especialmente perros. Aún más, el $52 \%$ de los que tenían perros indicaron que estos eran sus animales preferidos, seguido de las aves ornamentales $(36 \%)$. En el caso de los estudiantes, el $47 \%$ tenía al perro como único animal y el $47 \%$, además del perro, criaba otros animales (gatos, aves y peces ornamentales). Asimismo, el 93\% de estos jóvenes tenía al perro como animal preferido.

En el caso del grupo de responsables de animales de compañía, el $57 \%$ poseía perros, mientras que 12 y $9 \%$ poseían gatos y aves ornamentales, respectivamente. Asimismo, el 8\% compartía su vida con animales de varias especies, cuyo denominador común siempre era el perro. El preferido de este grupo resultó ser el perro (60\%), seguido por los gatos $(13 \%)$ y las aves ornamentales $(10 \%)$. En el estudio de Beovides et al. (2011) sobre tenencia de animales de compañía en otras ciudades cubanas se determinó, asimismo, que los animales preferidos eran los perros y gatos (24\% en ambos casos) en comparación con otras especies.

Estudios en Perú (Alva et al., 2010; Arévalo et al., 2010) y Chile (Abarca et al., 2011) registraron que la población objeto de estudio tenía perros y gatos en mayor proporción, seguidos por aves ornamentales, roedores, tortugas y animales exóticos (chimpancés y zorros); lo cual se corresponde con los hallazgos en las investigaciones cubanas (Beovides et al., 2011; Domínguez et al., 2011), excepto en la tenencia de animales exóticos, que no fue reportada en Cuba.

Se observó que la mayoría de los tenedores de animales de compañía los venían criando por 10 o más años. Asimismo, las respuestas sobre la satisfacción por la tenencia de animales de compañía concordaron en que estaban muy satisfechas o satisfechas al compartir sus vidas con animales de compañía (99\%). Un solo sujeto respondió que se sentía medianamente satisfecho (1\%).

Según refirió Elton (2003), el vínculo que el hombre ha creado en la actualidad con los animales de compañía va mucho más allá de una necesidad práctica y ha llegado a convertirse en una necesidad más bien emocional, lo cual se notó en los criterios expresados por las personas que formaron parte de este estudio.

\section{Beneficios sobre la Salud Psicoemocional}

Al indagar sobre la percepción que tenían las personas de estos colectivos sobre los beneficios que les podía reportar su relación con los animales de compañía (Cuadro 3 ), se encontró que la respuesta más frecuente fue de muy beneficiosa (84\% CAO, $73 \%$ E y $80 \%$ RAC). Únicamente un individuo planteó que solo le resultaba algo beneficioso. Por otro lado, fue significativo que una mujer que no podía tener hijos manifestó que la tenencia de su animal de compañía, un hámster, le prodigaba efectos muy beneficiosos sobre su salud mental, «ya que la distraía y no le hacía pensar en su problema (de no poder concebir)».

McConnell et al. (2011) encontraron que las personas que tenían animales de compañía eran menos propensas a los estados de depresión y aislamiento, y eran más agrada- 
Cuadro 2. Evaluación de la satisfacción personal en relación con la tenencia de animales de compañía (distribución porcentual)

\begin{tabular}{|c|c|c|c|}
\hline \multirow[b]{2}{*}{ Variable } & \multicolumn{3}{|c|}{ Grupo poblacional } \\
\hline & $\begin{array}{c}\text { Criadores de } \\
\text { aves } \\
\text { ornamentales } \\
\quad(n=25)\end{array}$ & $\begin{array}{c}\text { Estudiantes de } \\
\text { Medicina } \\
\text { Veterinaria } \\
(n=15)\end{array}$ & $\begin{array}{l}\text { Responsables } \\
\text { de animales de } \\
\text { compañía } \\
(\mathrm{n}=115)\end{array}$ \\
\hline \multicolumn{4}{|l|}{ Especie } \\
\hline Perro & - & 47 & 57 \\
\hline Gato & - & 6 & 12 \\
\hline Aves ornamentales & 44 & - & 9 \\
\hline Peces ornamentales & - & - & 6 \\
\hline Tortugas & - & - & 5 \\
\hline Roedores & - & - & 3 \\
\hline Otros & - & - & - \\
\hline Varias especies & 56 & 47 & 8 \\
\hline \multicolumn{4}{|l|}{ Animal de compañía preferido } \\
\hline Perro & 52 & 93 & 60 \\
\hline Gato & 4 & - & 13 \\
\hline Aves ornamentales & 36 & - & 10 \\
\hline Peces ornamentales & - & - & 4 \\
\hline Tortugas & - & - & 4 \\
\hline Roedores & - & - & 5 \\
\hline Otros & - & - & - \\
\hline Todos & 8 & 7 & 3 \\
\hline \multicolumn{4}{|l|}{ Tiempo de tenencia (en años) } \\
\hline Menos de 1 & 16 & 13 & 16 \\
\hline $1-3$ & 12 & 13 & 10 \\
\hline $4-6$ & 16 & - & 18 \\
\hline $7-9$ & 20 & 27 & 17 \\
\hline 10 o más & 36 & 47 & 38 \\
\hline \multicolumn{4}{|l|}{ Satisfacción de la tenencia } \\
\hline Muy satisfecho & 88 & 67 & 77 \\
\hline Satisfecho & 12 & 33 & 22 \\
\hline Medio satisfecho & - & - & 1 \\
\hline \multicolumn{4}{|l|}{ Relación con animales de compañía } \\
\hline Brinda compañía & 64 & 73 & 85 \\
\hline Brinda bienestar & 68 & 76 & 74 \\
\hline Libera tensiones & 36 & 33 & 83 \\
\hline Satisface contacto & 40 & 26 & 71 \\
\hline Mejora mi ánimo & 13 & 20 & 71 \\
\hline Me comunico con él & 28 & 46 & 61 \\
\hline Medio de comunicación familiar & 12 & 20 & 60 \\
\hline Cuido mejor de mi salud & 8 & 8 & 70 \\
\hline Compañero para mi soledad & 28 & - & 71 \\
\hline
\end{tabular}


Cuadro 3. Evaluación del beneficio de la relación entre las personas con sus animales de compañía (distribución porcentual)

\begin{tabular}{lccc}
\hline & \multicolumn{3}{c}{ Grupo poblacional } \\
\cline { 2 - 4 } Variable & $\begin{array}{c}\text { Criadores de } \\
\text { aves } \\
\text { ornamentales } \\
(\mathrm{n}=25)\end{array}$ & $\begin{array}{c}\text { Estudiantes de } \\
\text { Medicina } \\
\text { Veterinaria } \\
(\mathrm{n}=15)\end{array}$ & $\begin{array}{c}\text { Responsables } \\
\text { de animales de } \\
\text { compañía } \\
(\mathrm{n}=115)\end{array}$ \\
\hline Beneficios en la relación persona/animal & & & \\
$\quad$ Muy beneficiosa & 84 & 73 & 80 \\
$\quad$ Beneficiosa & 16 & 27 & 17 \\
$\quad$ Algo beneficiosa & - & - & 3 \\
Recomienda tenencia de animales de compañía & 88 & 100 & 78 \\
$\quad$ Sí & 12 & - & 15 \\
$\quad$ No & - & - & 7 \\
$\quad$ No sé & & & - \\
Mejora de la calidad de vida & 88 & - & 3 \\
$\quad$ Sí & 12 & - & 97 \\
No & - & & \\
$\quad$ No sé & & & \\
\hline
\end{tabular}

bles, extrovertidas y menos neuróticas que aquellas que no las tenían. Asimismo, las personas objeto del presente estudio manifestaron también los beneficios de los animales de compañía sobre su salud psicoemocional. Por otro lado, en el estudio realizado por Anzizu et al. (2001) en ancianos y trabajadores de una residencia asistida, se evidenció que los animales afectivos constituían una distracción (21\%) y un vehículo de expresión de afecto (18\%), principalmente para los ancianos.

La mayoría de los encuestados en los tres grupos (88\% CAO, $100 \%$ E y $78 \%$ RAC) recomendaron la tenencia de animales de compañía a otras personas. Sin embargo, las personas de mayor edad fueron más cautelosas al responder e hicieron razonamientos sobre el hecho de que había que tener en cuenta las condiciones de vivienda y económicas, así como sus necesidades afectivas y preferencias, para poder recomendar la tenencia de animales de compañía. Chur-
Hansen et al. (2008), en un estudio sobre la no tenencia de animales de compañía, identificaron que las razones expresadas con más frecuencia por personas de la tercera edad eran de índole emocional (relacionadas con la muerte del animal o su desamparo si el dueño fallecía, así como el hecho de tener familiares y no necesitar la compañía del animal) o pragmáticas (por no poder dedicarles tiempo).

$\mathrm{Al}$ examinar la contribución de la tenencia de animales de compañía en la mejora de la calidad de vida, la mayor parte de las respuestas fueron afirmativas ( $88 \%$ CAO, $100 \%$ E y $97 \%$ RAC).

\section{Beneficios sobre el Control Metabólico}

En el análisis sobre el padecimiento de enfermedades endocrinas y metabólicas (Cuadro 4), no se reportó el padecimiento de enfermedades de esta índole en el sector es- 
Cuadro 4. Evaluación de los aspectos benéficos de la tenencia de animales de compañía sobre el control metabólico (distribución porcentual)

\begin{tabular}{lccc}
\hline & \multicolumn{3}{c}{ Grupo poblacional } \\
\cline { 2 - 4 } Variable & $\begin{array}{c}\text { Criadores de } \\
\text { aves } \\
\text { ornamentales } \\
\text { (CAO) }\end{array}$ & $\begin{array}{c}\text { Estudiantes de } \\
\text { Medicina } \\
\text { Veterinaria } \\
(\text { E) }\end{array}$ & $\begin{array}{c}\text { Responsables } \\
\text { de animales de } \\
\text { compañía } \\
\text { (RAC) }\end{array}$ \\
\hline Padece enfermedades endocrinas o metabólicas & & \\
Diabetes mellitus & 12 & - & 13 \\
Obesidad & - & - & 16 \\
Lípidos elevados & - & - & 14 \\
Tiroides & 4 & - & 5 \\
Infertilidad & 8 & - & 28 \\
No padece & 53 & 73 & 16 \\
Otras & 24 & 27 & - \\
Efecto del cumplimiento del tratamiento & & & 60 \\
Me quita tiempo & 12 & - & 64 \\
Motiva una mejor nutrición & 16 & - & - \\
Motiva a hacer ejercicios & 48 & 75 & 7 \\
Me cuido más & 58 & 25 & \\
Hacer test de Benedict en orina & 12 & - & \\
No efecto & - & - & \\
Otras & & &
\end{tabular}

${ }^{1}$ CAO, $n=25 ; E, n=15 ; R A C, n=115$

tudiantil. En los otros grupos, las principales enfermedades fueron la obesidad, diabetes mellitus (DM) e hiperlipidemias.

En la valoración de la motivación del cumplimiento del tratamiento de las enfermedades que padecían por convivir con un animal de compañía, las respuestas más frecuentes fueron «me cuido más» $(58 \% \mathrm{CAO}, 96 \%$ RAC) y «me motiva a hacer ejercicios» $(48 \%$ CAO, 64\% RAC). Para las mujeres fue más importante el hecho de cuidarse más, sobre todo para las de más edad, que expresaron que se cuidaban porque sentían que si les faltaban a sus animales, estos podrían sufrir y quedar desamparados. En el caso del grupo $\mathrm{RAC}$, el $60 \%$ indicó que tener un animal de compañía los motivaba a tener una mejor nutrición, lo cual implicaba cuidados para su salud física y control metabólico, tanto en hombres como en mujeres.

En los individuos que padecían DM se indagó sobre los posibles cambios de conducta que podían advertir en los animales de compañía cuando manifestaban síntomas de hipo o hiperglucemia. Diez de 18 personas declararon que observaban cambios de conducta en sus animales (perros y gatos) desde el comienzo de los síntomas, ya que se ponían nerviosos, ladraban o maullaban, cambiaban su comportamiento y no dejaban de estar a su lado y se mostraban más atentos. Cambios conductuales en los perros al advertir episodios de hipoglucemia en sus dueños han sido reportados por Wells et al. (2008). Por 
Cuadro 5. Evaluación de la motivación para la realización de la actividad física en relación con la tenencia de animales de compañía (distribución porcentual)

\begin{tabular}{|c|c|c|c|}
\hline & $\begin{array}{l}\text { Criadores de } \\
\text { aves } \\
\text { ornamentales } \\
\quad(\mathrm{CAO})\end{array}$ & $\begin{array}{l}\text { Estudiantes de } \\
\text { Medicina } \\
\text { Veterinaria } \\
\text { (E) }\end{array}$ & $\begin{array}{c}\text { Responsables } \\
\text { de animales } \\
\text { de compañía } \\
\text { (RAC) }\end{array}$ \\
\hline Caminatas con animales de compañía & $(n=14)$ & $(n=14)$ & $(n=74)$ \\
\hline Sí & 50 & 64 & 53 \\
\hline No realiza caminatas & $(n=7)$ & $(n=5)$ & $(n=35)$ \\
\hline Falta de ánimo & 43 & - & 9 \\
\hline Limitaciones & 43 & - & 11 \\
\hline No le gusta salir con el animal & - & 20 & 3 \\
\hline Peligro para el animal & 57 & - & 40 \\
\hline Peligro para la persona & 43 & - & 11 \\
\hline Animal agresivo & - & - & 9 \\
\hline Prefiere que otros lo paseen & - & - & 9 \\
\hline Otras causas & - & 80 & 20 \\
\hline Frecuencia de la caminata & $(n=7)$ & $(n=9)$ & $(n=39)$ \\
\hline $1-2 /$ mes & - & 22 & 12 \\
\hline $1 /$ semana & 14 & 22 & 12 \\
\hline 2-3/semana & - & - & 18 \\
\hline$+4 /$ semana & 29 & 22 & 20 \\
\hline Todos los días & 57 & 34 & 38 \\
\hline Tiempo de las caminatas (min) & $(n=7)$ & $(n=9)$ & $(n=39)$ \\
\hline $15-30$ & 72 & 55 & 74 \\
\hline $30-60$ & 14 & 34 & 20 \\
\hline$>60$ & 14 & 11 & 6 \\
\hline Efecto de las caminatas & $(n=7)$ & $(n=9)$ & $(n=39)$ \\
\hline Disminuye el peso corporal & 43 & - & 64 \\
\hline Ayuda al control metabólico & 29 & - & 51 \\
\hline Promueve caminatas & 57 & - & 64 \\
\hline Disfruto de su compañía & 100 & 89 & 94 \\
\hline Me mantiene saludable & 86 & 100 & 97 \\
\hline Me produce relajación & 72 & 78 & 94 \\
\hline Estímulo para realizar ejercicios & 72 & 89 & 90 \\
\hline Es una rutina & 29 & 22 & 74 \\
\hline Ejercicios para el animal & - & - & 51 \\
\hline Otras & 29 & 55 & 12 \\
\hline
\end{tabular}

otro lado, tres personas no advirtieron cambios conductuales en sus perros y cinco de ellas no pudieron advertirlo pues solo tenían hámsteres, tortugas y peces ornamentales.
$\mathrm{Al}$ preguntar si la tenencia de animales de compañía contribuía de manera beneficiosa en el control de las enfermedades que padecían, se obtuvo una respuesta afirmati- 
va (75\% CAO, 72\% RAC). Asimismo, al preguntarle a las personas con DM que habían notado los cambios en la conducta de sus perros si estos cambios los motivaba a tomar cuidados, 7 de 10 personas afirmaron que trataban de ajustar su tratamiento y se auto-monitoreaban más seguido. Estos resultados denotaron que la tenencia de estos animales puede ser un aliciente para que las personas practiquen el autocuidado, lo cual ejerce un efecto beneficioso sobre su salud mental y física.

\section{Motivaciones para la Actividad Física}

$\mathrm{Al}$ evaluarse las motivaciones para realizar actividad física (Cuadro 5), se pudo advertir que entre el 50 y $64 \%$ de los tenedores de perros salían a caminar con ellos. Las personas que no lo hacían en los grupos CAO y RAC sentían que podía haber un peligro para el animal. Por otro lado, entre aquellos que realizaban las caminatas, la mayor frecuencia era diaria y por periodos de 15 a 30 minutos.

El principal efecto positivo de las caminatas fue la percepción de que los mantiene saludables en el caso de los estudiantes, y en el disfrute de la compañía de los animales en el caso del grupo CAO. Por otro lado, estas dos respuestas fueron las más frecuentes en el caso del grupo RAC; asimismo, también indicaron que les provocaba un efecto relajante y les estimulaba a hacer ejercicios.

Resultados de investigación demuestran que los animales afectivos constituyen un estímulo para realizar caminatas (Anzizu et al., 2001). Por otro lado, Motooka et al. (2006) encontraron que personas ancianas que paseaban con sus perros obtenían mayor beneficio para su salud en comparación con aquellas que caminaban sin ellos, lo cual fue corroborado por McConnell et al. (2011). Asimismo, González y Landero (2011) evaluaron aspectos relacionados con el estrés, la salud mental y física de los seres humanos y observaron que las personas que considera- ban a sus perros como miembros de la familia, realizaban con ellos actividades con mayor frecuencia.

Beck (1997) planteó que algunos de los beneficios sociales más importantes de la interacción humano-animal eran el efecto catalizador -lubricante social-, la cohesión social, el juego cooperativo, la mayor cooperación con otras personas y la promoción del contacto social interpersonal entre los miembros de la familia. Algunos de estos aspectos fueron percibidos por los encuestados.

\section{Conclusiones}

Las personas encuestadas en los tres grupos poblacionales perciben que la tenencia de animales de compañía aporta beneficios a la salud de las personas que cohabitan con estos animales.

\section{Literatura Citada}

1. Abarca K, López del P J, Peña A, López J. 2011. Tenencia y estado de salud de mascotas de niños inmunocomprometidos, con énfasis en enfermedades zoonóticas. Rev Chil Infect 28: 205-210.

2. Alva R, Revilla E, Isla C. 2010. Convivencia con animales de compañía en niños y jóvenes estudiantes del distrito de Chiclayo, provincia de Chiclayo, Lambayeque-Perú. En: XXII Congreso Panamericano de Ciencias Veterinarias. Lima.

3. Anzizu J, Botella L, Carles J. 2001. Evaluación de una terapia asistida por animales de compañía (TAAC) en un colectivo de ancianos institucionalizados a partir del análisis del discurso de los usuarios. [Internet], [18 enero 12]. Disponible en: http://www.psiquiatria.com/ neuropsiquiatria/psicogeriatria/ evaluacion-de-una-terapia-asistida-por- 
animales-de-compania-taac-en-un-colectivo-de-ancianos-institucionalizadosa-partir-del-analisis-del-discurso-de-losusuarios/

4. Arévalo W, Rachumi V, Eslava J. 2010. Tenencia responsable de animales de compañía en niños y jóvenes estudiantes del distrito de Ferreñafe, Lambayeque-Perú. En: XXII Congreso Panamericano de Ciencias Veterinarias. Lima.

5. Beck AM. 1997. La relación de las personas mayores: compañía, salud y calidad de vida. III Congreso Internacional El Hombre y los Animales de Compañía. Barcelona: Fundación Purina. p 17-32.

6. Beovides LM, Silveira PE, Norman MO. 2011. Evaluación del cuidado de la salud de mascotas en el municipio Santa Clara, Cuba. En: VII Congreso Internacional de Ciencias Veterinarias. La Habana.

7. Chur-Hansen A, Winefield $H$, Beckwith M. 2008. Reasons given by elderly men and women for not owning a pet, and the implications for clinical practice and research. J Health Psychol 13:988-995.

8. Cusack, O. 1991. Animales de compañía y salud mental. Barcelona: Fundación Purina. 256 p.

9. Domínguez H, Pérez Y, Barrios V. 2011 Características de la tenencia de la población felina en la ciudad de Matanzas. En: VII Congreso Internacional de Ciencias Veterinarias. La Habana.

10. Elton ME. 2003. Diseño de una pauta de trabajo para la aplicación de terapia asistida con perros. Tesis de Médico Veterinario. Valdivia: Universidad Austral de Chile. 91 p.

11. Gatti RM. 1994. Mascotas y ancianos. Efectos mascota positivos. Bol Vet Sipavet 10(4): 10-14.

12. González MT, Landero R. 2011. Diferencias en estrés percibido, salud mental y física de acuerdo al tipo de relación humano-perro. Rev Colomb Psicol 20(1): 75-86.

13. McConnellAR, Brown CM, Shoda TM, Stayton LE, Martin CE. 2011. Friends with benefits: on the positive consequences of pet ownership. J Pers Soc Psychol 101: 1239-1252.

14. Mentzel R. 2004. Origen y evolución del vínculo humano-animal. Rev Med Vet 85: 139-145.

15. Motooka Koike H, Yokoyama T, Kennedy NL. 2006. Effect of dogwalking on autonomic nervous activity in senior citizens. Med J Aust 184: 60-63.

16. Staats, S, Sears K, Pierfelice L. 2006. Teachers' pets and why they have them: An investigation of the human-animal bond. J Appl Soc Psychol 36: 1881-1891.

17. Wells DL, Lawson SW, Siriwardena N. 2008. Canine responses to hypoglycemia in patients with type 1 diabetes. J Altern Complement Med 14: 1235-1241. 